\title{
A Parechovirus Type 3 Infection with a Presumed Intrauterine Onset: A Poor Neurodevelopmental Outcome
}

\author{
Sahar Salavati ${ }^{a}$ Masoud Salavati $^{\text {b, c }}$ Maraike A. Coenen ${ }^{d}$ Hendrik J. ter Horst ${ }^{a}$ \\ Arend F. Bos ${ }^{\mathrm{a}}$ \\ ${ }^{a}$ Division of Neonatology, Department of Pediatrics, Beatrix Children's Hospital, University Medical Center \\ Groningen, University of Groningen, Groningen, The Netherlands; ${ }^{b}$ Royal Dutch Visio, Center of Expertise for \\ Blind and Visually Impaired People, Huizen, The Netherlands; ${ }^{C}$ Healthy Ageing, Allied Health Care and Nursing, \\ Hanzehogeschool Groningen, University of Groningen, University Medical Center Groningen, Groningen, \\ The Netherlands; ${ }^{d}$ Department of Clinical Neuropsychology, University of Groningen, University Medical Center \\ Groningen, Groningen, The Netherlands
}

\section{Established Facts}

- Parechovirus type $3(\mathrm{HPeV}-3)$ infection is an important cause of illness in neonates.

- Symptoms can range from a mild gastroenteritis to sepsis and meningoencephalitis with possibly neurological sequela.

- Most HPeV-3 infections occur in the neonatal period.

\section{Novel Insights}

- HPeV-3 infection may occur already in utero.

- Intrauterine onset of the $\mathrm{HPeV}-3$ infection is related to a poor neurodevelopmental outcome, and therefore timely recognition is important.

\section{Keywords}

Parechovirus type $3 \cdot$ Intrauterine infection .

Neurodevelopment

\section{Abstract}

Parechovirus type 3 (HPeV-3) infection is an important cause of illness in neonates. We present the first case of an infant with a HPeV-3 meningoencephalitis which presumably commenced in utero. Severe developmental delay was seen. In the case of inexplicable neonatal meningoencephalitis, an intrauterine onset of $\mathrm{HPeV}-3$ infection might be the cause.

(c) 2020 The Author(s)

Published by S. Karger AG, Basel

\section{Introduction}

Parechovirus type $3(\mathrm{HPeV}-3)$ infections are increasingly being recognized as an important cause of infections in neonates and infants [1]. The first case of $\mathrm{HPeV}$ -

C 2020 The Author(s)

Published by S. Karger AG, Basel

This article is licensed under the Creative Commons AttributionNonCommercial-NoDerivatives 4.0 International License (CC BYNC-ND) (http://www.karger.com/Services/OpenAccessLicense). Usage and distribution for commercial purposes as well as any distribution of modified material requires written permission.
Sahar Salavati

Division of Neonatology, Department of Pediatrics, Beatrix Children's Hospital University Medical Center Groningen, University of Groningen

Hanzeplein 1 HPC CA51, NL-9713GZ Groningen (The Netherlands)

s.salavati@umcg.nl 
Fig. 1. Cerebral ultrasound imaging showing increased periventricular echodensity (day 2) and bilateral cystic evolution (day

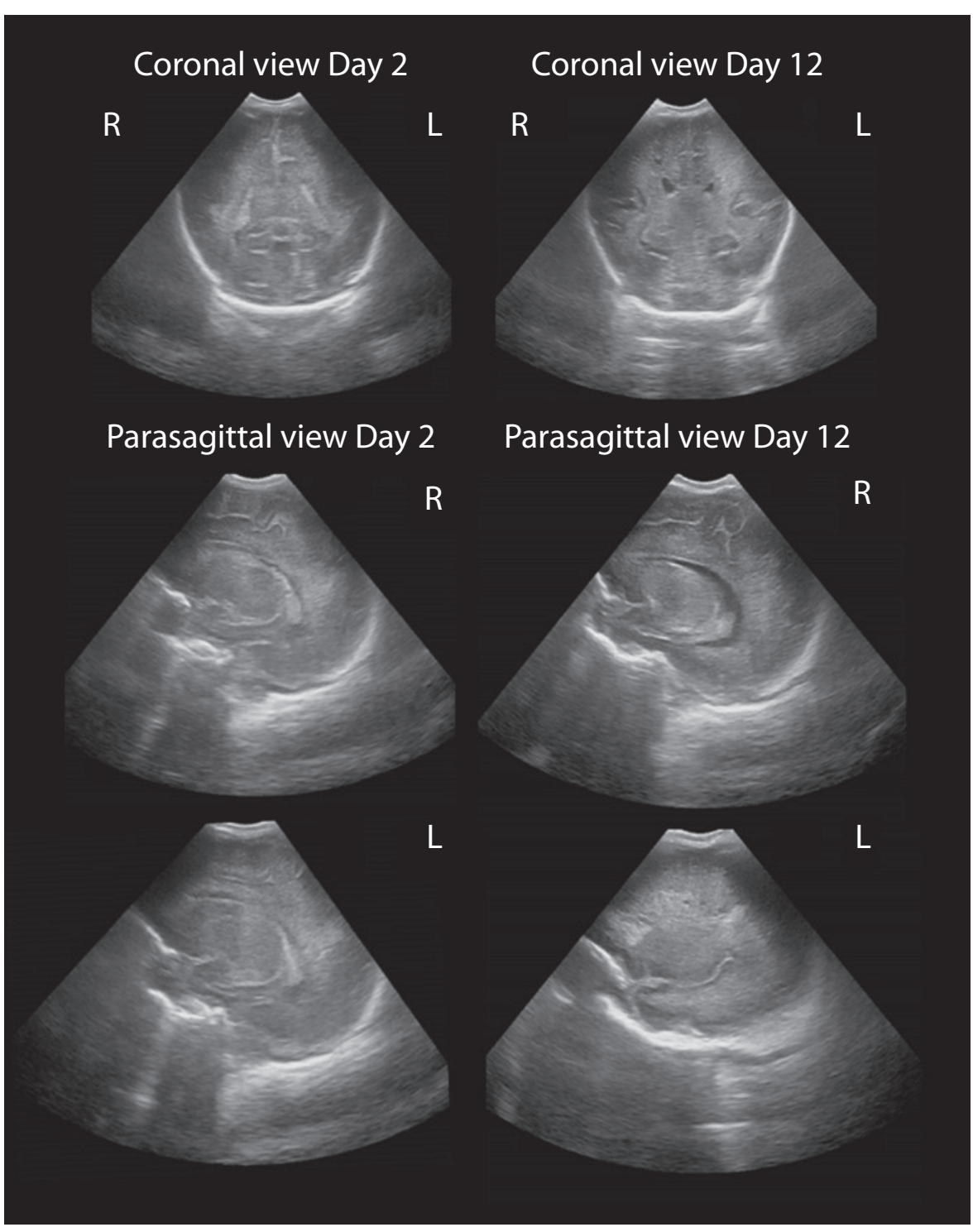
12).

3 was reported in 2004 and described a 1-year-old girl in Japan who presented with transient paralysis and a high fever [2]. Since then, several outbreaks of $\mathrm{HPeV}-3$ have been reported. Symptoms can range from mild gastroenteritis to sepsis and meningoencephalitis with possibly neurological sequela. The presence of maternal antibodies seems to play a protective role in the pathogenesis [3].

Most HPeV-3 infections occur in the neonatal period [3]. Only 1 case of maternal HPeV-3 infection has been reported, but without vertical transmission [4]. To our knowledge, no studies exist on intrauterine infections with $\mathrm{HPeV}-3$.

Our aim was to report the neurodevelopmental trajectory of an infant infected with $\mathrm{HPeV}-3$, presumably in utero. This case report generates the hypothesis that intrauterine onset of the HPeV-3 infection is related to poor neurodevelopmental outcomes and therefore timely recognition is important.

\section{Case Report}

A boy was born at a gestational age of 32 weeks and 4 days by emergency cesarean section. This was performed because of reduced fetal movements since a few days and the absence of baseline heart rate variability on cardiotocography. He weighed 2,347 g $(+0.79 \mathrm{SD})$ and had a head circumference of $31.1 \mathrm{~cm}(+0.60 \mathrm{SD})$. Apgar scores were 7, 4, and 6 after 1, 5, and $10 \mathrm{~min}$, respectively. Umbilical cord pH values were 7.37 (arterial) and 7.40 (venous). Soon after birth the infant became bradycardic and pale, and he was 


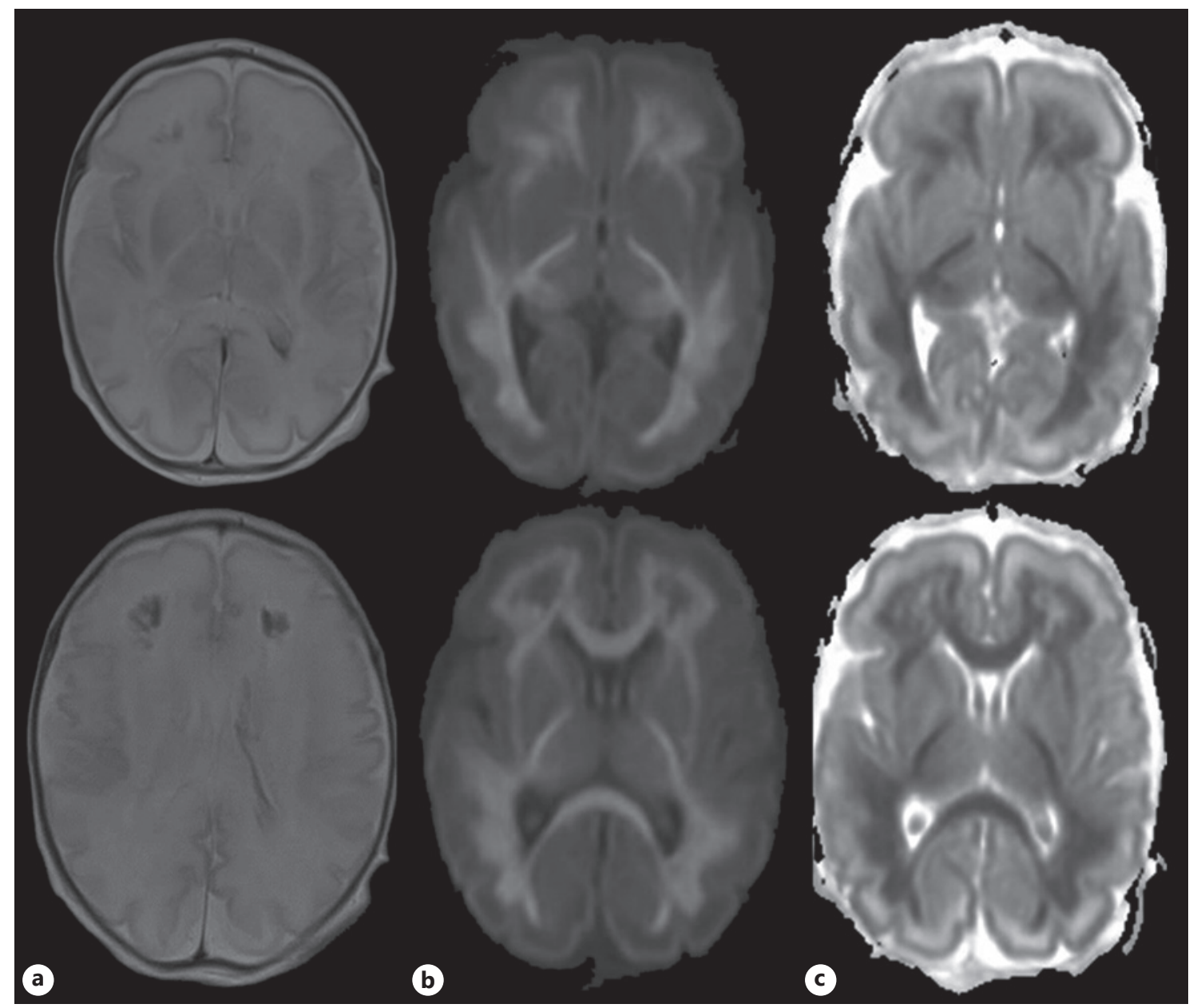

Fig. 2. T2-weighted MRI (a), diffusion weighted image (b) with apparent diffusion coefficient map (c) images at a postnatal age of 5 days showing extensive bilateral ischemic injury with restricted diffusion in the white matter, the corpus callosum, the corticospinal tract, the pulvinar, and optic radiation. Furthermore, hemorrhagic changes within the distribution of the medullary veins in the frontal lobes and left-sided intraventricular hemorrhage are seen.

hypotonic and hypokinetic. Respiration was insufficient, and therefore he was intubated and ventilated for 5 days. He received a single dose of surfactant. Physical examination, on day 1 , showed a greyto-pink, reactive but hypokinetic infant with evident hypotonia.

Initially, the cause of fetal distress was not fully understood. There was no clear history of perinatal asphyxia. Lactate levels were low $(1.6 \mathrm{mmol} / \mathrm{L})$. Liver tests and renal function were normal. The CRP on days 0 and 1 was $<5 \mathrm{mg} / \mathrm{L}$. As perinatal bacterial infection could not be ruled out, treatment with broad-spectrum antibiotics was started immediately after birth and ceased after $48 \mathrm{~h}$ because of negative cultures. In the first days after birth the boy remained hypotonic and hypokinetic. The mother's medical history revealed that she had suffered a mild gastroenteritis 1-2 weeks before delivery. The family history was negative for inborn errors of metabolism. Based on the mother's medical history, additional viral tests were performed. $\mathrm{HPeV}-3 \mathrm{RNA}$ was detected in both feces (genotyping) and cerebrospinal fluid (CSF; using PCR). The samples were collected on days 3 and 5 after birth, respectively.
Additionally, CSF pleocytosis was present (leucocytes: $197 \times$ $10^{6} / \mathrm{L}$ ). The aEEG on day 3 showed a low-voltage background pattern (burst suppression) with subclinical seizures, for which the infant received phenobarbital and midazolam. Repetitive cerebral ultrasound imaging demonstrated cystic evolution. Already on day 2 after birth, increased periventricular echodensity was seen and cysts were present from day 12 (Fig. 1). No additional risk factors for the development of periventricular leukomalacia were present. Furthermore, magnetic resonance imaging (MRI) on day 5 demonstrated extensive bilateral ischemic injury with restricted diffusion in the white matter, the corpus callosum, the corticospinal tract, the pulvinar, and optic radiation. There were hemorrhagic changes within the distribution of the medullary veins in the frontal lobes, which were confirmed by susceptibility weighted imaging. In addition, a left-sided intraventricular hemorrhage was present (Fig. 2). These findings are suggestive of a viral encephalitis. Both serial Doppler ultrasound imaging and MRI showed no signs of venous thrombosis. 
Genetic testing and tests for inborn errors of metabolism were normal.

The treatment of parechovirus meningoencephalitis comprised supportive care.

\section{General Movements Trajectory}

The infant's general movements (GMs) were assessed in order to monitor his early neurological status from birth until 14 weeks' corrected age [5]. Over time, the GMs deteriorated from poor repertoire to cramped synchronized movements and ultimately to the absence of fidgety movements.

\section{Neurological Development at 2.5 Years of Age}

Between 2 and 2.8 years the boy underwent several examinations and tests, including physical examination and assessments of his vision and motor, cognitive, language, and behavioral development.

At a corrected age of 2 years and 1 month the boy had a head circumference of $45.7 \mathrm{~cm}(-2$ to $-2.5 \mathrm{SD})$. At a calendar age of 2 years and 6 months the boy was diagnosed with bilateral spastic cerebral palsy (CP) with a Gross Motor Function Classification System (GMFCS) grade IV-V. Using Visual Impairment-Adapted Gross Motor Function Measure-88 (GMFM-88-CVI) a total score of $24 \%$ was found [6]. At a calendar age of 2 years and 8 months the boy was diagnosed with cerebral visual impairment (CVI) based on visual functional assessments.

At a calendar age of 2 years and 9 months cognitive and language development were assessed using the Bayley Scales of Infant and Toddler Development-Third Edition-The Netherlands-Special Needs Addition (Bayley-III-NL-SNA). Index scores for cognition and language were 58 (percentile 0.6) and 60 (percentile 1.6), respectively.

Behavior and emotional problems were assessed at 2 years' corrected age using the parental questionnaire Child Behavior Checklist (CBCL). Scores were indicative of behavioral problems (T score total problems: 67, clinical range), with emphasis on internalizing behavioral problems (71, clinical range). These internalizing problems were characterized by problems with emotion regulation and withdrawn behavior. In addition, the score for attention problems was clinically elevated.

Written informed consent was obtained from both parents.

\section{Discussion/Conclusion}

Increasingly, $\mathrm{HPeV}-3$ infections are being recognized as a cause of illness in neonates. Clinical manifestations and sequela vary, but they may cause serious neonatal illness and neurodevelopmental problems later on [7].

Some aspects of the present case with presumed intrauterine transmission are in line with previous reports on postnatally acquired neonatal $\mathrm{HPeV}-3$ infections. These include the clinical manifestations with meningoencephalitis, and MRI findings of white matter injury [1]. White matter abnormalities reported in $\mathrm{HPeV}-3$ resemble the abnormalities caused by hypoxic ischemic encephalopathy. It has been suggested that the severity of MRI findings is indicative of poor neurodevelopmental outcomes [7].

The uniqueness of this case lies in the intrauterine onset of the disease, highly likely based on the history of the mother and clinical signs existing already before birth, mimicking perinatal asphyxia. It has some similarities with an antenatal echovirus type 6 infection which causes severe meningoencephalitis and eventually death [8]. All reported HPeV-3 infections so far have occurred postnatally. Newborns are probably more susceptible to a more severe course of the disease because of a low seroprevalence of $\mathrm{HPeV}-3$ in women of childbearing age. In our case, the nonspecific clinical course during the first days after birth following presumed asphyxia raised suspicion of another etiology of the clinical course. Additional diagnostics into genetic, metabolic, and viral causes were performed during these first days and revealed $\mathrm{HPeV}-3$ in infantile feces and CSF. Even though no certainty on the timing of infection exists, we consider an intrauterine onset of an $\mathrm{HPeV}-3$ infection to be the most likely cause of illness in this child.

The neurodevelopmental outcome of this child was at the most severe end of the spectrum of outcomes reported thus far [1]. The severity of the neurodevelopmental delay may be explained by the combination of meningoencephalitis and prematurity. Both processes can lead to activation of microglia cells and damage of oligodendrocytes, the latter impeding myelination.

The early ultrasound and MRI abnormalities of this infant asked for close monitoring of his early development. Both the presence of cramped synchronized GMs and the absence of fidgety movements are strong indicators of the development of CP [9]. Recently, GMs were also reported to be predictive of the neurodevelopment of infants infected with the Zika virus in utero [10]. As this case shows, combining MRI with GMs can further improve the prognostic value for outcome [9].

The developmental domains we tested and that were affected in this child covered a wide range. Visual and motor impairment were CVI and CP, which are relatively often seen in conjunction [6]. To date, only CP but not CVI has been reported in children with $\mathrm{HPeV}-3$ infections. Only Britton et al. [7] reported a case of neonatal infection with an unknown type of $\mathrm{HPeV}$ that was diagnosed with CVI. A proper visual examination could be a point of attention in these children.

The cognitive, language, and behavioral problems of the boy in our report might be the direct result of brain 
damage due to $\mathrm{HPeV}-3$ meningoencephalitis, although both CP and CVI can impede normal development in these domains [6]. It is therefore difficult to distinguish, in a test setting, whether delayed development is primary or secondary to CP or CVI or to both. We tried, however, to minimize potential negative effects of $\mathrm{CP}$ and CVI on test results by using the Bayley-III-NL-SNA, which is suited and validated for children with special needs.

This case report demonstrates the poor neurodevelopmental course following an HPeV-3 infection that most likely occurred in utero. The boy was preterm born because of suspected fetal distress, diagnosed postnatally with meningoencephalitis, and his GMs were increasingly abnormal over time and consistent with poor visual, motor, and neuropsychological functioning up to the age of 2.8 years.

We strongly recommend that health workers consider the possibility of an intrauterine infection with $\mathrm{HPeV}-3$ in infants with sepsis-like illnesses and inexplicable signs of fetal distress. As indicated in this report, a detailed medical history remains the backbone of sound medical practice and, in addition to neuroimaging, it emphasizes the value of the assessment of GMs as a tool for early prediction of neurodevelopmental outcomes.

\section{Acknowledgement}

We greatly acknowledge the help of Dr. Titia Brantsma with correcting the English language of this work.

\section{Statement of Ethics}

Written informed consent for publication of the case (including imaging) was obtained from both parents.

\section{Conflict of Interest Statement}

The authors have no conflict of interests relevant to this article to disclose.

\section{Funding Sources}

No funding was secured for this study.

\section{Author Contributions}

Prof. Bos and Ms. Salavati conceptualized and designed this study, drafted the initial version of this paper, and reviewed and revised this paper. Ms. Coenen and Dr. Salavati designed the data collection instruments, collected the data, and reviewed and revised this paper. Dr. ter Horst interpreted the clinical data and critically reviewed this paper for important intellectual content. All of the authors approved the final version of this paper as submitted and agree to be accountable for all aspects of this work.

\section{References}

1 Verboon-Maciolek MA, Groenendaal F, Hahn CD, Hellmann J, van Loon AM, Boivin $\mathrm{G}$, et al. Human parechovirus causes encephalitis with white matter injury in neonates. Ann Neurol. 2008 Sep;64(3):266-73.

2 Ito M, Yamashita T, Tsuzuki H, Takeda N, Sakae K. Isolation and identification of a novel human parechovirus. J Gen Virol. 2004 Feb; 85(Pt 2):391-8.

3 Harvala H, Robertson I, Chieochansin T, McWilliam Leitch EC, Templeton K, Simmonds P. Specific association of human parechovirus type 3 with sepsis and fever in young infants, as identified by direct typing of cerebrospinal fluid samples. J Infect Dis. 2009 Jun;199(12): 1753-60.

4 Shinomoto M, Kawasaki T, Sugahara T, Nakata $\mathrm{K}$, Kotani T, Yoshitake H, et al. First report of human parechovirus type 3 infection in a pregnant woman. Int J Infect Dis. 2017 Jun;59:22-4.
5 Einspieler C, Marschik PB, Pansy J, Scheuchenegger A, Krieber M, Yang H, et al. The general movement optimality score: a detailed assessment of general movements during preterm and term age. Dev Med Child Neurol. 2016 Apr;58(4):361-8.

6 Salavati M, Rameckers EA, Waninge A, Krijnen WP, Steenbergen B, van der Schans CP. Gross motor function in children with spastic cerebral palsy and cerebral visual impairment: a comparison between outcomes of the original and the Cerebral Visual Impairment adapted Gross Motor Function Measure-88 (GMFM-88-CVI). Res Dev Disabil. 2017 Jan; 60:269-76.

7 Britton PN, Dale RC, Nissen MD, Crawford N, Elliott E, Macartney K, et al.; PAEDS-ACE Investigators. Parechovirus encephalitis and neurodevelopmental outcomes. Pediatrics. 2016 Feb;137(2):e20152848.
8 van den Berg-van de Glind GJ, de Vries JJ, Wolthers KC, Wiggers-de Bruine FT, PeetersScholte CM, van den Hende M, et al. A fatal course of neonatal meningo-encephalitis. J Clin Virol. 2012 Oct;55(2):91-4.

9 Novak I, Morgan C, Adde L, Blackman J, Boyd RN, Brunstrom-Hernandez J, et al. Early, accurate diagnosis and early intervention in cerebral palsy: advances in diagnosis and treatment. JAMA Pediatr. 2017 Sep;171(9): 897-907.

10 Einspieler C, Utsch F, Brasil P, Panvequio Aizawa CY, Peyton C, Hydee Hasue R, et al.; GM Zika Working Group. Association of Infants Exposed to Prenatal Zika Virus Infection With Their Clinical, Neurologic, and Developmental Status Evaluated via the General Movement Assessment Tool. JAMA Netw Open. 2019 Jan;2(1):e187235. 